were rated as having moderate language delay, an outcome that is less readily interpreted than severe delay. Children producing fairly complete sentences were rated as having no language delay. Among a total of 38,954 children, $204(0.5 \%)$ had severe language delay. Of the reference group $(24 \%)$ who took no dietary supplements, $81(0.9 \%)$ had severe language delay. (In Norway, foods are not fortified with folic acid).

Relative risks of severe language delay by estimating odds ratios for 3 patterns of exposure to folic acid were (1) $0.9 \%$ [OR 1.04] in children of mothers taking other supplements but no folic acid; (2) $0.4 \%$ [0.55] for those taking folic acid only; and (3) $0.4 \%[0.55]$ for those taking folic acid and other supplements. The adjusted ORs and risk of moderate language delay for the three groups were $1.04,0.82$, and 0.79 , respectively. Maternal use of folic acid supplements in early pregnancy, 4 weeks before to 8 weeks post-conception, was associated with a reduced risk of severe language delay in children at age 3 years. Maternal reports and Vineland test scores for receptive and expressive communication skills in a subsample of 425 children were highly correlative. A higher proportion of children with severe language delay had not yet attained expected motor skills at 18 months and 3 years, but use of folic acid supplements was not correlated with attainment of gross motor skills. (Roth C, Magnus P, Schjolberg S, et al. Folic acid supplements in pregnancy and severe language delay in children. JAMA October 12, 2011;306(14):1566-1573). (Respond: Christine Roth MSc ClinPsyD, Division of Mental Health, Norwegian Institute of Public Health, PO Box 4404, Nydalen, 0403 Oslo, Norway. E-mail: Christine.roth@fhi.no).

COMMENT. Periconceptional folic acid supplements are known to reduce risk of neural tube defects (MRC Vitamin Study. Lancet 1991;338(8760):131-137), but the present study is considered the first to demonstrate effects on language development. The average child usually says his first word by 1 year, and delay beyond 18 months may indicate a severe physical, mental or hearing handicap. Failure to put 2 or 3 words together in short phrases by 2 years and sentences by age 3 years is a significant delay in language development. Adequate hearing is the first requisite for normal language development. If the hearing is normal, the differential diagnosis includes congenital dysphasia, mental retardation, and infantile autism. The brain MRI, fMRI (de Guibert C et al. Brain 2011;134:3044-3058) or pathological studies (Cohen M et al. Ann Neurol 1989;25:567-570) may uncover subtle cerebral anomalies. An EEG may be indicated to rule out an acquired epileptic aphasia (Landau-Kleffner syndrome) that develops at 2 to 5 years of age.(Cockerell I et al. Epilepsy Behav 2011;21:153-159) The cause of severe language delay associated with periconceptional folic acid deficiency is unclear. Prenatal effects on brain development (Milunsky A et al. JAMA 1989;262:2847-2852), and epigenetic dysregulation of gene expression are proposed possible mechanisms.. (Jaenisch R et al. Nat Genet 2003;33(suppl):245-254).

\title{
ABNORMAL FUNCTIONAL LATERALIZATION OF LANGUAGE IN DEVELOPMENTAL DYSPHASIA
}

Researchers at University Hospital, Rennes, France compared functional MRI studies of 21 children with developmental dysphasia and matched controls using a panel of four language tests including auditory and visual tasks. Children with specific 
language impairment exhibited a significant lack of left lateralization in core language regions (inferior frontal gyrus-opercularis, inferior frontal gyrus-triangularis, supramarginal gyrus and superior temporal gyrus). Between group comparisons revealed a left hypoactivation of Wernicke's area during the responsive naming task, and a right hyperactivation of the anterior insula, inferior frontal gyrus and head of caudate during a phonological task. Developmental dysphasia is associated with atypical lateralization and functioning of core language areas. (de Guibert C, Maumet C, Jannin P et al. Abnormal functional lateralization and activity of language brain areas in typical specific language impairment (developmental dysphasia). Brain 2011;134:3044-3058). (Respond: Dr Clement de Guibert, Sciences du langage, Universite Rennes 2, Place H Le Moal, CS 24307, F-35043 Rennes Cedex, France. E-mail: clement.deguibert@univ-rennes2.fr).

COMMENT. The etiology of specific language impairment is unclear; an interaction between genes and environmental risk factors may affect the anatamofunctional development and organization of the brain language network (Rapin I et al, 2003). fMRI and other studies show that an abnormality of brain development is bilateral and not confined to the left hemisphere.

\section{INFANTILE ENCEPHALOPATHIES}

\section{KCNQ2 ENCEPHALOPATHY AND NEONATAL SEIZURES}

Researchers from centers in Belgium and Australia analyzed 80 patients with unexplained neonatal or early infantile seizures and associated psychomotor retardation for KCNQ2 mutations. Seven different heterozygous $K C N Q 2$ mutations were found in eight patients $(8 / 80 ; 10 \%)$; six mutations arose de novo. No KCNQ3 mutations were found. The eight $K C N Q 2$ encephalopathy patients had onset of intractable seizures with tonic component in the first week of life. Seizures resolved by age 3 years but the children had profound or severe psychomotor impairment. EEG at onset showed a multifocal epileptiform activity. Early brain MRI showed hyperintensities in the basal ganglia and thalamus that later resolved. $K C N Q 2$ screening should be included in the diagnostic workup of refractory neonatal seizures of unknown origin. (Weckhuysen S, Mandelstam S, S!!ls A, et al. KCNQ2 encephalopathy: Emerging phenotype of a neonatal epileptic encephalopathy. Ann Neurol Oct 2011;published on line, 10.1002/ana 22644).(Respond: Prof Peter De Jonghe, University of Antwerp, Belgium. E-mail: peter.dejonghe@molgen.vib-ua.be).

COMMENT. Mutations in the voltage gated $\mathrm{K}(+)$-channel gene $K C N Q 2$ cause benign familial neonatal convulsions, the majority having a favorable outcome. (Singh NA et al. Brain 2003;126:2726-2737) Reports of patients with a poor outcome are infrequent. Dedek K and associates (Epilepsy Res 2003;54(1):21-27) of Hamburg, Germany reported two children in an Italian family with neonatal convulsions and $K C N Q 2$ mutations, the index patient having a poor outcome and therapy-resistant epilepsy. The present report is supportive of the association of some $K C N Q 2$ mutations with an infantile epileptic encephalopathy complicated by psychomotor impairment and refractory seizures. 